\title{
Habilidades sociales y la educación virtual de estudiantes universitarios
}

\section{Social skills and virtual education of university students}

1 Karla Daniela Villacís Pico

https://orcid.org/0000-0002-7510-677X

Universidad Técnica de Ambato, Carrera de Psicopedagogía, Ambato, Tungurahua,

Ecuador.

kvillacis4941@uta.edu.ec

2 Leticia Abigail Mayorga Ases

https://orcid.org/0000-0003-0586-2390

Universidad Técnica de Ambato, Facultad Ingeniería Civil y Mecánica. Ingeniería

Mecánica,

la.mayorga@uta.edu.ec

3 María José Mayorga Ases

https://orcid.org/0000-0003-1897-739X

Universidad Técnica de Ambato, Carrera de Psicopedagogía, Ambato, Tungurahua,

Ecuador.

mariajmayorga@uta.edu.ec

4 Katty Patricia Sánchez Manobanda ID https://orcid.org/0000-0001-5390-209X

Universidad Técnica de Ambato, Carrera de Psicopedagogía, Tungurahua,

kp.sanchez@uta.edu.ec

Artículo de Investigación Científica y Tecnológica

Enviado: 24/12/2021

Revisado: $29 / 12 / 2021$

Aceptado: $12 / 01 / 2022$

Publicado:08/03/2023

DOI: https://doi.org/10.33262/concienciadigital.v6i1.4.2037

Cítese: Villacís Pico, K. D., Mayorga Ases, L. A., Mayorga Ases, M. J., \& Sánchez Manobanda, K. P. (2023). Habilidades sociales y la educación virtual de estudiantes universitarios. ConcienciaDigital, $6(1.4)$, https://doi.org/10.33262/concienciadigital.v6i1.4.2037 862-879.

CONCIENCIA DIGITAL, es una Revista Multidisciplinar, Trimestral, que se publicará en soporte electrónico tiene como misión contribuir a la formación de profesionales competentes con visión humanística y crítica que sean capaces de exponer sus resultados investigativos y científicos en la misma medida que se promueva mediante su intervención cambios positivos en la sociedad. https://concienciadigital.org .

La revista es editada por la Editorial Ciencia Digital (Editorial de prestigio registrada en la Cámara Ecuatoriana de Libro con No de Afiliación 663) www.celibro.org.ec 
Palabras

claves:

habilidades

sociales,

educación

virtual,

modalidad

virtual, educación en covid-19.
Keywords:

social skills,

virtual

education,

virtual

modality, education in covid-19.
Resumen

Introducción. En la actualidad la educación se ha visto obligada a dar un giro de 360 grados, pues gracias a la pandemia por el virus COVID-19, desde el 16 de marzo del 2020 la educación presencial se vio obligada a transformarse en educación virtual. Con ello las relaciones sociales que se consolidaban por medio de la educación también se vieron afectadas, de modo que los estudiantes tuvieron que crear nuevas habilidades para socializar. Objetivo. Identificar la influencia de la educación virtual en las habilidades sociales de los estudiantes universitarios de la Universidad Técnica de Ambato.

Metodología. Por medio de una investigación de tipo descriptiva con enfoque mixto realizada a 105 estudiantes de la Universidad Técnica de Ambato, quienes respondieron a un test sobre las Habilidades sociales y una encuesta sobre la educación virtual. Resultados. Se obtuvo una correlación de Spearman de 0.757 entra la variable de Habilidades sociales y la educación virtual. Conclusión. Los estudiantes a quienes se les aplicó las dos encuestas evidencian que la educación virtual si ha afectado significativamente sus habilidades sociales, lo cual es un punto que tratar por parte de las instituciones de educación superior juntamente con los docentes y estudiantes, creando métodos o estrategias que incentiven las habilidades sociales en la educación virtual.

\section{Abstract}

Introduction. At present, education has been forced to take a 360degree turn, because thanks to the pandemic caused by the COVID19 virus, since March 16, 2020, face-to-face education was forced to transform into virtual education. With this, the social relationships that were consolidated through education were also affected, so that the students had to create new skills to socialize. Target. Identify the influence of virtual education on the social skills of university students at the Technical University of Ambato.

Methodology. Through descriptive research with a mixed approach carried out on 105 students from the Technical University of Ambato, who responded to a test on social skills and a survey on virtual education. Results. A Spearman correlation of 0.757 was obtained between the variable of social skills and virtual education. Conclusion. The students to whom the two surveys were applied show that virtual education has significantly affected their social 
skills, which is a point to be addressed by higher education institutions together with teachers and students, creating methods or strategies that encourage social skills in virtual education.

\section{Introducción}

\section{Habilidades Sociales}

Los seres humanos son entes sociales por naturaleza, pues independientemente del ámbito y el contexto en el que se encuentren, desde la infancia crean habilidades sociales para relacionarse. Hay quienes consideran que las habilidades sociales son conductas que se aprenden y se refuerzan a lo largo de la vida conforme el ser humano se desarrolla y el contexto en el cual se desenvuelve la mayor parte de su vida. Con las habilidades sociales los seres humanos expresan sentimientos, deseos, opiniones y derechos adecuándose a las conductas de sus pares, dando una respuesta inmediata a los problemas que se presentan en la actualidad y minimizando futuros problemas (Maximina et al., 2021).

\section{Tabla 1}

Componentes de las habilidades sociales

\begin{tabular}{cl}
\hline Componentes & \multicolumn{1}{c}{ Descripción } \\
\hline Verbales & $\begin{array}{l}\text { El tono y el timbre de voz, la fluidez, la velocidad al hablar y la } \\
\text { gesticulación que utiliza una persona a la hora de entablar una conversación } \\
\text { con otra. }\end{array}$ \\
& $\begin{array}{l}\text { La caligrafía, la ortografía, el lenguaje corporal y el contacto visual son } \\
\text { factores para tomar en cuenta dentro de las habilidades sociales no } \\
\text { verbales. }\end{array}$ \\
\hline
\end{tabular}

\section{Fuente: Morales (2018)}

Las habilidades sociales son un conjunto de hábitos que nos permiten mejorar nuestras relaciones interpersonales sentirnos bien obtener lo que queremos y conseguir que los demás no nos impidan lograr nuestros objetivos es la capacidad de relacionarnos con los demás en forma tal que consigamos un máximo de beneficios y un mínimo de consecuencias negativas tanto a corto como a largo plazo esto incluye temas afines como asertividad autoestima y la inteligencia emocional Asimismo las habilidades sociales influyen en la percepción en la que el adolescente tiene de sí mismo de los otros de la sociedad y que le permite responder de manera positiva ante situaciones de estrés (Coronel, 2019).

A la hora de interactuar y relacionarse el ser humano buscar hacerlo en su mayoría de forma afectiva y satisfactoria mediante conductas como el comportamiento interpersonal, el diálogo, la participación, la confianza, la escucha activa, la formulación de preguntas, 
las emociones y deseos que reflejan las habilidades sociales de cada persona en el contexto que se desenvuelva un individuo (Núñez y Zambrano, 2021).

Los niños y adolescentes están en constante cambio desde contextos y ambientes sociales, lo que los hace susceptibles a asimilar la forma en la que se relacionan conforme sus pares y en los adultos con los que conviven, la familia y la escuela son ámbitos de aprendizaje de habilidades sociales. Los adolescentes buscan constantemente pertenecer y ser aceptados en un grupo social, poniendo en práctica las habilidades sociales que adquieren mediante los valores y comportamientos que se exponen en los medios de comunicación que acostumbran a observar, el clima familiar en que se desenvuelven, su inteligencia emocional, la autoestima, el uso ya sea correcto o incorrecto que le dan a las redes sociales y la institución educativa a la que pertenecen (Montes et al., 2018).

\section{Tabla 2}

\section{Modelo Jerárquico}

\begin{tabular}{ll}
\hline \multicolumn{1}{c}{ Componentes } & \multicolumn{1}{c}{ Descripción } \\
\hline $\begin{array}{l}\text { Conducta prosocial versus } \\
\text { antisocial }\end{array}$ & $\begin{array}{l}\text { El polo positivo es el autocontrol y las relaciones sociales; el polo } \\
\text { negativo es la agresividad. }\end{array}$ \\
$\begin{array}{l}\text { Sociabilidad versus baja } \\
\text { socialización }\end{array}$ & $\begin{array}{l}\text { En esta dimensión predomina el liderazgo desde lo positivo y en lo } \\
\text { negativo se evidencia el bajo control de las relaciones sociales, } \\
\text { ansiedad social y el retraimiento. }\end{array}$ \\
\hline
\end{tabular}

Fuente: Coronel et al. (2020)

\section{Habilidades sociales en la educación superior}

La educación es un proceso social en el cual los actores directos son los docentes y los estudiantes, lo que significa que son precursores de esquemas de socialización y comunicación que desarrolla habilidades sociales que repercuten en el aprendizaje, no obstante cabe mencionar que gracias a la modalidad de educación virtual los estudiantes y docentes se han visto afectados y una de las repercusiones es el retraso de las habilidades sociales, de modo que la tecnología genera un cambio de actitud y en ocasiones hasta de mentalidad debido a la calidad de relaciones sociales que en esta se forjan (Tixi et al., 2020).

En el ámbito educativo las habilidades sociales juegan un papel muy importante, puesto que existe una relación positiva entre las habilidades sociales y los logros escolares, sin embargo, el escaso manejo de habilidades sociales en los estudiantes crea dificultades como la poca o nula aceptación, lo que conlleva a un aislamiento y problemas emociones. Estas dificultades en el ambiente escolar originan que el estudiante mucho más allá de tener problemas en el ámbito académico tenga problemas en lo psicológico y este es un factor crucial en el aprendizaje, pues un estudiante consolida su aprendizaje de mejor 
manera cuando se encuentra estable física, emocional y psicológicamente (Salas et al., 2020).

Es por esto por lo que, diseñar e implementar estrategias que promuevan las habilidades sociales debe convertirse en un compromiso de toda institución educativa y al referirnos a estas no se puede pasar por alto que las maneras de pensar y de actuar de los jóvenes son influenciadas por sus metas, siendo éstas una fuente motivacional que los docentes y padres deben conocer, ya que puede ser adecuadas influyendo positivamente en el comportamiento interpersonal. Al no ser adecuadas es necesario determinar por qué la persona afronta la actividad social como lo hace, para ayudarla a modificar dichas metas si es necesario (Gil y Andreu, 2020).

No obstante, la compleja sociedad en la que vivimos requiere una formación escolar que trascienda el simple aprendizaje de contenidos académicos, razón por la cual es necesario comprender que las habilidades sociales, como aptitud a fortalecer en los centros educativos,

los cuales deben convertirse en su principal riqueza, razón por la cual la comunicación asertiva y demás elementos que promuevan este tipo de conductas socialmente habilidosas se privilegien, se gestionen y promuevan en las instituciones del país, pues la responsabilidad principal de mantener un clima organizacional apropiado recae en primer lugar sobre cada centro educativo, Cabe mencionar, que la conducta socialmente habilidosa es ese conjunto de conductas expresadas por una persona en un contexto interpersonal, que devela los sentimientos, deseos, opiniones, aptitudes, de un modo armónico, que facilita la resolución de problemas futuros (Horna-Clavo et al., 2020).

Comúnmente los adolescentes que están cursando la universidad aún se encuentran forjando su identidad, enfrentándose a desafíos natos de la adolescencia y acogiéndose a su vez de la aceptación social para resolverlos. Hay quienes consideran que las habilidades sociales se consolidan en su mayoría en la adolescencia y el paso por la universidad, desembocando en que las habilidades no cognitivas influyen el ámbito laboral de una persona, gracias a que si una persona cuenta con un buen manejo de sus habilidades sociales puede conseguir un buen empleo, permanecer en él y hasta sobresalir (Botello et al., 2020).

A pesar de que hay estudiantes ejemplares por su rendimiento académico que terminan su carrera universitaria con reconocimientos por sus calificaciones, en el ámbito laboral tienen dificultades por el nulo manejo de las habilidades sociales. Es por esto varias universidades se enfocan en crear programas de intervención en habilidades sociales, que fomenten el trabajo en equipo, la comunicación asertiva, la empatía y el respeto hacia los demás, es decir las habilidades sociales básicas (Cárdenas \& Inacio, 2018). 
En la actualidad la responsabilidad de la educación recae en la calidad del docente, porque las estrategias, técnicas y métodos que use para impartir el conocimiento a sus estudiantes de manera virtual es un reto, y mucho más aún incentivar a los estudiantes a que formen relaciones sociales y potencien sus habilidades, pues la educación no trata únicamente de lo cognitivo, sino implica los factores sociales, culturales, económicos, etc. Esto conlleva que el docente impulse a los estudiantes a trabajar en equipo, a interactuar dentro y fuera del horario de clases. Pues el contexto educativo actual no facilita la consolidación de nuevas habilidades sociales.

\section{La educación virtual}

La educación virtual o como también se la conoce "educación en línea", transforma la educación convencional presencial a un escenario de enseñanza-aprendizaje en el ciberespacio; esta es una estrategia que busca mejorar la calidad educativa, mediante la adquisición de conocimientos a través del uso de las Tecnologías de la Información y Comunicación (TIC), posibilitando a los estudiantes crear nuevos hábitos de estudio que involucren contextos de aprendizaje en la red y estos a su vez consoliden el aprendizaje de una manera más personalizada (Crisol et al., 2020).

La educación virtual ha ido creciendo poco a poco, trazando parámetros que ponen en práctica actividades virtuales eficaces y eficientes, en las cuales las personas involucradas con intereses diferentes, pero con un objetivo similar, logran exponer y consolidar el conocimiento a partir de un intercambio de saberes, experiencias e ideas, en un proceso formativo. Para desarrollar una educación virtual de calidad hace falta que el estudiante asuma la responsabilidad de mediar con su tiempo disponible y la predisposición a aprender de una manera más independiente, es decir buscando alternativas investigativas que le permitan consolidar y reforzar su aprendizaje (García, 2019).

Los jóvenes y niños nacidos entre los años 1995 y 2015 son conocidos como la "Generación Z", estos son denominados como "nativos digitales", ya que han crecido conforme la tecnología se ha ido desarrollando, por lo que las TIC han formado parte de su vida y darles uso ya sea para educarse, recrearse, informarse, etc., es demasiado sencillo, no obstante hay hogares en los que los padres no son participes de mantener a sus hijos en constante contacto con la tecnología y otros hogares no cuentan con los recursos económicos para hacerlo. Este también fue un beneficio en el momento en que la educación se transformó a lo virtual, pues quienes ya dominaban las TIC no tuvieron mayor problema que instalar o descargar programas, por otra parte, también hay estudiantes que debieron salir a buscar un dispositivo electrónico para recibir sus clases virtuales y aprender a usarlo (Juca et al., 2020). 


\section{Educación virtual en Ecuador}

En Ecuador a causa de la pandemia de COVID-19, desde el 16 de marzo del 2020, las instituciones educativas fiscales, privadas y de educación superior, se han acogido a la modalidad de teletrabajo, el cual se rige al plan educativo Covid-19. Esto, para la mayoría de las personas que estaban acostumbradas a la modalidad presencial, fue controversial ya que el adaptarse a la virtualidad genero frustración y miedo a fracasar. Además de limitar la convivencia entre el estudiante y el docente a medios digitales, como aplicaciones de video conferencia y mensajería, convirtiendo estos en la nueva aula de clase (Cáceres, 2020).

La UNICEF menciona que en Ecuador solo el 37\% de las familias tienen acceso a internet, dejando a 6 de cada 10 niños despojados de la posibilidad de continuar con sus estudios mediante plataformas digitales. Haciendo que para los niños en zonas rurales sea más difícil mantenerse en el sistema educativo, ya que solo el 16\% de los hogares cuentan con acceso a este servicio. Al contrario 9 de cada 10 hogares cuentan con televisor, teléfono celular o radio, lo que evidencia una vía para poder llegar a través de estos medios y que los niños y jóvenes continúen aprendiendo (UNICEF, 2020).

De igual forma el currículo nacional que estaba vigente desde el 2016 también fue remplazado por el currículo priorizado para la emergencia. En el cual los objetivos de aprendizaje se enlazan con los objetivos integradores de cada año o nivel, igualmente las destrezas con criterio de desempeño se amoldan a conceptos esenciales y al desarrollo de habilidades de pensamiento, valores y la contención emocional que para esta época de cuarentena es muy necesaria. De manera que con estas modificaciones el gobierno espera que los jóvenes no sean excluidos, permanezcan en el sistema educativo y cuenten con una educación prioritaria (Ministerio de Educación Ecuador [MINEDUC], 2020).

En la actualidad los espacios virtuales de educación son dinámicos pero variantes, pues van transmutando en benéfico del aprendizaje cooperativo, gracias a que en este predomina el monólogo del profesor sin embargo se transforma en un proceso de enseñanza interactivo. A pesar de esto la virtualidad no remplaza ni representa la realidad de lo que sucede en lo presencial, más bien es una simulación de esta, a la que no todas las clases sociales pueden acceder, generando una exclusión en la educación (Pérez et al., 2018)

Es por esto por lo que la virtualidad también se ha vuelto una desventaja para algunos estudiantes, que gracias a la crisis económica que la pandemia generó, es limitado el acceso a algunos servicios básicos, como el internet o la electricidad, que son indispensables para acceder a una educación virtual, lo que los pone en desventaja para adquirir el aprendizaje. Por ende, es fundamental que los docentes consideren nuevas dinámicas o estrategias en las que se puedan dar alternativas de solución a las necesidades 
de los estudiantes (Potesta et al., 2021).

Por lo cual se han identificado tres brechas en el mundo digital:

Tabla 3

Brechas de desigualdad en la Educación Virtual

\begin{tabular}{ll}
\hline \multicolumn{1}{c}{ Componentes } & Descripción \\
\hline Limitaciones económicas & $\begin{array}{l}\text { Hace referencia a la falta de acceso a un dispositivo } \\
\text { tecnológico por escases de recursos económicos. }\end{array}$ \\
Limitaciones de acceso & $\begin{array}{l}\text { Se evidencia la incapacidad para la adquisición de } \\
\text { dispositivos tecnológicos, sin embargo, no se puede } \\
\text { cubrir el pago mensual de un servicio de internet. }\end{array}$ \\
Limitaciones económicas y de acceso & $\begin{array}{l}\text { Es nada más que la suma de las dos brechas anteriores se } \\
\text { evidencia } \\
\text { con mayo frecuencia en los sectores rurales y gracias a } \\
\text { esto los estudiantes no pueden dar continuidad a su } \\
\text { educación. }\end{array}$ \\
\hline
\end{tabular}

Fuente: Cobo et al. (2020)

De modo que la tecnología educativa debe ser más humanista en el proceso de aprendizaje y acoplarse a las situaciones de los estudiantes y profesores, esto con el objetivo de facilitar la labor docente y mejorar el rendimiento académico. Los docentes son quienes deben fomentar la iniciativa, la atención y participación de los estudiantes de manera individual y colectiva, brindando constantemente su guía para ir forjando el camino hacia el aprendizaje con metodologías que busquen dar solución a conflictos de manera razonable, para que a pesar de la distancia física el estudiante entienda que el docente más que una representación de autoridad es un ente de apoyo que facilite las situaciones de crisis educativa (Cueva, 2020).

\section{La educación virtual en las universidades}

En la educación superior se implementó el uso de las TIC en las universidades del Ecuador, puesto que esto es uno de los requisitos para la acreditación de carreras y universidades, sin embargo, en el momento en el que la educación se transformó obligatoriamente a la modalidad virtual, se pudo evidenciar que no todos los docentes y estudiantes tenían conocimiento del manejo de herramientas tecnológicas que permitan dar esta continuidad a la educación. Esto evidenció que más que nada los docentes necesitan estar en constante preparación con cursos, charlas etc., en los que se instruya sobre cómo utilizar herramientas tecnológicas y como crear un ambiente de estudio más dinámico (Sousa et al., 2021). 
Investigaciones a estudiantes universitarios tras la pandemia por COVID-19, postulan que estos son más propensos a desarrollar ansiedad, estrés y depresión, debido a las situaciones económicas, educativas y sociales. En estudiantes chinos se evidenció un elevado índice de síntomas depresivos, estrés post traumático y trastornos del sueño; otro estudio en estudiantes griegos delimitó un gran número de estudiantes con pensamientos suicidas en la población. Estos resultados son preocupantes ya que la salud mental es un punto muy serio que requiere de atención de las universidades (Cobo et al., 2020).

Varias universidades alrededor del continente americano han creado mecanismos de apoyo psicológico y socioemocional principalmente para los estudiantes, fortaleciendo el servicio del bienestar estudiantil con fuentes de apoyo psicológico en tiempos de pandemia, que ofrecen ayuda para enfrentar la angustia, la ansiedad, el estrés, el duelo, entre otro factos psicológicos que afectan la salud mental de los estudiantes universitarios, sin embargo al ver la gran demanda de personas que no pertenecían a estas universidades buscar ayuda en estos departamentos, la mayoría de estas ofertan este servicio gratuito a la sociedad, lo que beneficia también a los estudiantes de carreras relacionadas con el trato de estos problemas y que están prontos a adquirir su título universitario, pues son estos quienes ponen en práctica sus conocimientos en estos sitios de ayuda (Instituto Internacional para la Educación Superior [IESAlC], 2020).

El clima escolar también sufrió un cambio brusco, puesto que cambió el contexto escolar presencial a la virtualidad, lo que inmiscuyó a que el clima escolar se contraste en su mayoría con la familia, por lo tanto los padres de familia están encargados de verificar que sus hijo cuenten con la conexión a internet adecuada para dar pie a sus clases virtuales, cumplan con el horario establecido por las instituciones, proveer el material necesario para el desarrollo de las actividades académicas y brindar motivación y apoyo que evite que los estudiantes tengan una deserción escolar (Gavilánez et al., 2021).

Por otra parte, los docentes son ejes fundamentales en la educación y mucho más aun en la modalidad virtual, estos se han visto en la obligación de implementar estrategias de apoyo para estudiantes en pandemia. Aplican las tutorías entre pares para fomentar el vínculo de cercanía con los estudiantes a pesar del distanciamiento físico, tutorías de apoyo en la materia que se ha brindado con anterioridad, espacios en los que se puede hacer consultas al docente y este brinda orientación a los estudiantes, talleres relacionados con el tema a tratar y de manera muy sutil abordan apoyo psicológico, sin embargo, es de una forma indirecta (Coca et al., 2021).

\section{Metodología}

El presente artículo es de tipo descriptivo con un enfoque mixto, puesto que su objetivo es el análisis de la correlación que existe entre las habilidades sociales y la educación virtual en estudiantes universitarios; la recolección de datos se realizó mediante la 
aplicación de dos encuestas a una muestra de 105 estudiantes de la Universidad Técnica de Ambato, el primer cuestionario aplicado fue el de habilidades sociales "En busca de mi crecimiento personal" y el segundo cuestionario en educación virtual, a los cuales se aplicó la correlación de Spearman. Los resultados de estas encuestas sirven para verificar si existe una relación entre las habilidades sociales y a la educación virtual, ya sean estas positivas o negativas.

\section{Resultados:}

Se procede aplicar el test de habilidades sociales en una muestra de 105 estudiantes de las diferentes carreras de la Facultad de Ciencias Humanas la muestra se realizó de forma aleatoria, en la cual se obtuvieron los siguientes resultados.

\section{Tabla 4}

Respuestas del test de habilidades sociales "En busca de mi crecimiento personal"

\begin{tabular}{|c|c|c|c|c|c|c|c|c|c|c|}
\hline Nro. & $\begin{array}{c}\text { Totalmente } \\
\text { en } \\
\text { desacuerdo }\end{array}$ & $\%$ & $\begin{array}{c}\text { En } \\
\text { desacuerdo }\end{array}$ & $\%$ & $\begin{array}{c}\text { Ni de } \\
\text { acuerdo ni } \\
\text { en } \\
\text { desacuerdo }\end{array}$ & $\%$ & $\begin{array}{c}\text { De } \\
\text { acuerdo }\end{array}$ & $\%$ & $\begin{array}{l}\text { Totalmente } \\
\text { de acuerdo }\end{array}$ & $\%$ \\
\hline 1 & 3 & $2,86 \%$ & 9 & $8,57 \%$ & 22 & $20,95 \%$ & 52 & $49,52 \%$ & 19 & $18,10 \%$ \\
\hline 2 & 5 & $4,76 \%$ & 19 & $18,10 \%$ & 25 & $23,81 \%$ & 47 & $44,76 \%$ & 9 & $8,57 \%$ \\
\hline 3 & 5 & $4,76 \%$ & 2 & $1,90 \%$ & 19 & $18,10 \%$ & 48 & $45,71 \%$ & 31 & $29,52 \%$ \\
\hline 4 & 4 & $3,81 \%$ & 3 & $2,86 \%$ & 19 & $18,10 \%$ & 54 & $51,43 \%$ & 25 & $23,81 \%$ \\
\hline 5 & 4 & $3,81 \%$ & 6 & $5,71 \%$ & 3 & $2,86 \%$ & 48 & $45,71 \%$ & 44 & $41,90 \%$ \\
\hline 6 & 29 & $27,62 \%$ & 33 & $31,43 \%$ & 21 & $20,00 \%$ & 13 & $12,38 \%$ & 9 & $8,57 \%$ \\
\hline 7 & 3 & $2,86 \%$ & 1 & $0,95 \%$ & 2 & $1,90 \%$ & 29 & $27,62 \%$ & 70 & $66,67 \%$ \\
\hline 8 & 59 & $56,19 \%$ & 28 & $26,67 \%$ & 13 & $12,38 \%$ & 4 & $3,81 \%$ & 1 & $0,95 \%$ \\
\hline 9 & 36 & $34,29 \%$ & 13 & $12,38 \%$ & 18 & $17,14 \%$ & 31 & $29,52 \%$ & 7 & $6,67 \%$ \\
\hline 10 & 1 & $0,95 \%$ & 2 & $1,90 \%$ & 14 & $13,33 \%$ & 43 & $40,95 \%$ & 45 & $42,86 \%$ \\
\hline 11 & 38 & $36,19 \%$ & 36 & $34,29 \%$ & 23 & $21,90 \%$ & 7 & $6,67 \%$ & 1 & $0,95 \%$ \\
\hline 12 & 1 & $0,95 \%$ & 1 & $0,95 \%$ & 10 & $9,52 \%$ & 39 & $37,14 \%$ & 54 & $51,43 \%$ \\
\hline 13 & 7 & $6,67 \%$ & 5 & $4,76 \%$ & 13 & $12,38 \%$ & 29 & $27,62 \%$ & 51 & $48,57 \%$ \\
\hline 14 & 3 & $2,86 \%$ & 6 & $5,71 \%$ & 27 & $25,71 \%$ & 44 & $41,90 \%$ & 25 & $23,81 \%$ \\
\hline 15 & 7 & $6,67 \%$ & 37 & $35,24 \%$ & 23 & $21,90 \%$ & 26 & $24,76 \%$ & 12 & $11,43 \%$ \\
\hline 16 & 12 & $11,43 \%$ & 14 & $13,33 \%$ & 38 & $36,19 \%$ & 29 & $27,62 \%$ & 12 & $11,43 \%$ \\
\hline 17 & 2 & $1,90 \%$ & 28 & $26,67 \%$ & 5 & $4,76 \%$ & 20 & $19,05 \%$ & 50 & $47,62 \%$ \\
\hline 18 & 3 & $2,86 \%$ & 1 & $0,95 \%$ & 16 & $15,24 \%$ & 48 & $45,71 \%$ & 37 & $35,24 \%$ \\
\hline
\end{tabular}

Fuente: Estudio de contextos estudiantes Universidad Técnica de Ambato

Tras aplicar a 105 estudiantes de la Universidad Técnica de Ambato el test de habilidades sociales "En busca de mi crecimiento personal", conformado por 18 preguntas de 5 opciones de respuesta en escala de Likert totalmente en desacuerdo, en desacuerdo, ni de acuerdo ni en desacuerdo, de acuerdo y totalmente de acuerdo, se obtuvieron los porcentajes establecidos en la tabla 4 , donde se puede ver que existe un mayor porcentaje 
en que los estudiantes se encuentran de acuerdo en que ha existido un desequilibrio en las actualidad en sus habilidades sociales, influenciado por la pandemia del Covid-19 y por la virtualización de las clases que no permite una relación fluida la cual se daba de manera presencial.

Después de tomar el test de habilidades sociales se procede a realizar la encuesta de educación virtual previamente validada por alfa de Cronbach obteniendo en una muestra de 50 personas un valor de 0.978 . Se procede a tomarla en los 105 estudiantes que fueron seleccionados de forma aleatoria de la Facultad de Ciencias Humanas y de la Educación de las distintas carreras que tiene la Facultad.

Tabla 5

Respuestas de la encuesta de educación virtual

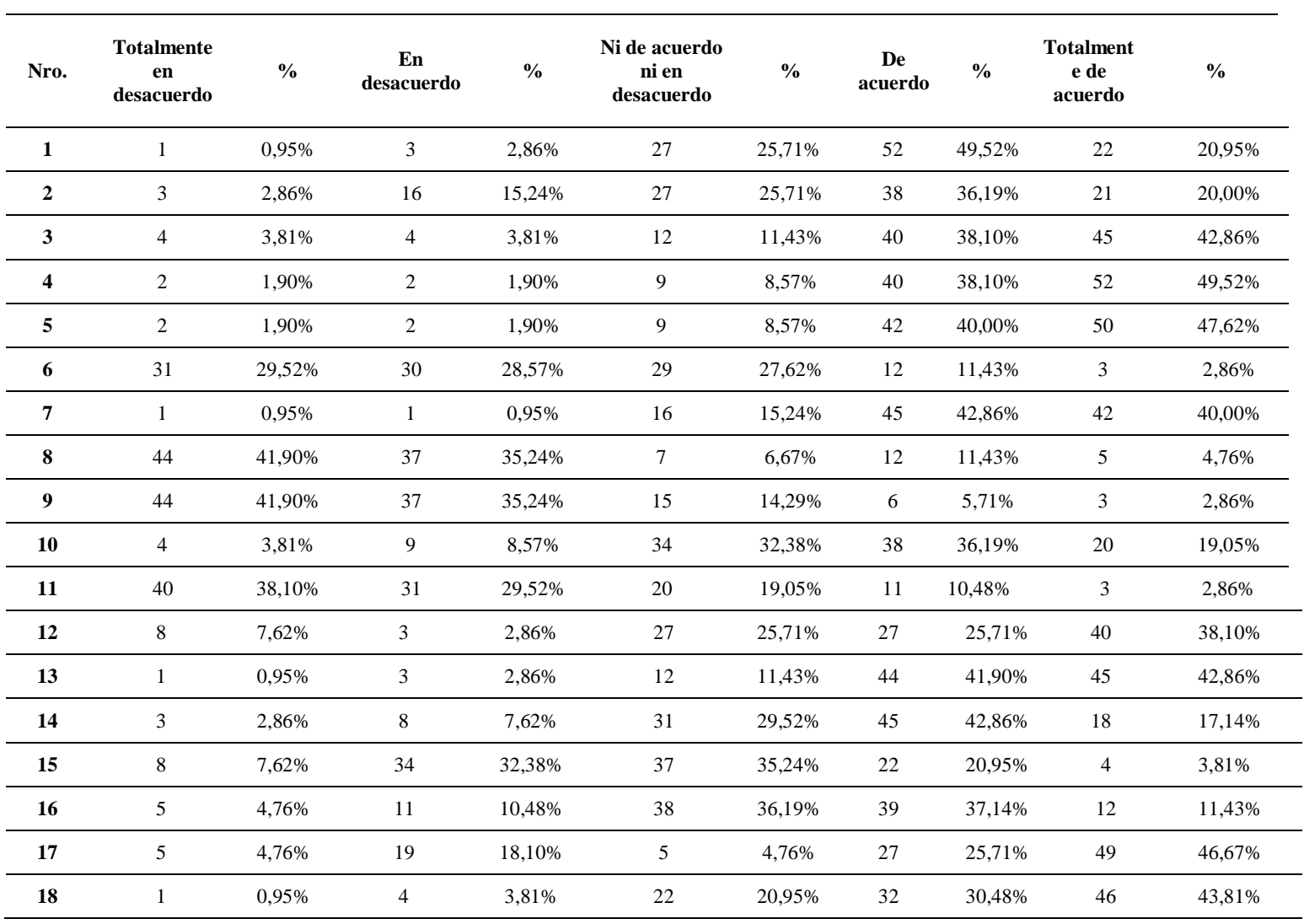

Fuente: Estudio de contextos estudiantes Universidad Técnica de Ambato

Los resultados de la encuesta de educación virtual muestran que hay un mayor porcentaje en la escala de Likert de acuerdo, lo que significa que la mayoría de los estudiantes han tenido dificultades en la adaptación a la Educación Virtual al inicio de la pandemia, los mismos se dan por problemas de conexión en el internet, la falta de recursos tecnológicos, y la adaptación a una educación virtual de manera repentina, lo cual dificulta el trato con sus pares. 


\section{Figura 1}

Correlación de Spearman de los resultados de las encuestas de Habilidades Sociales y Educación Virtual

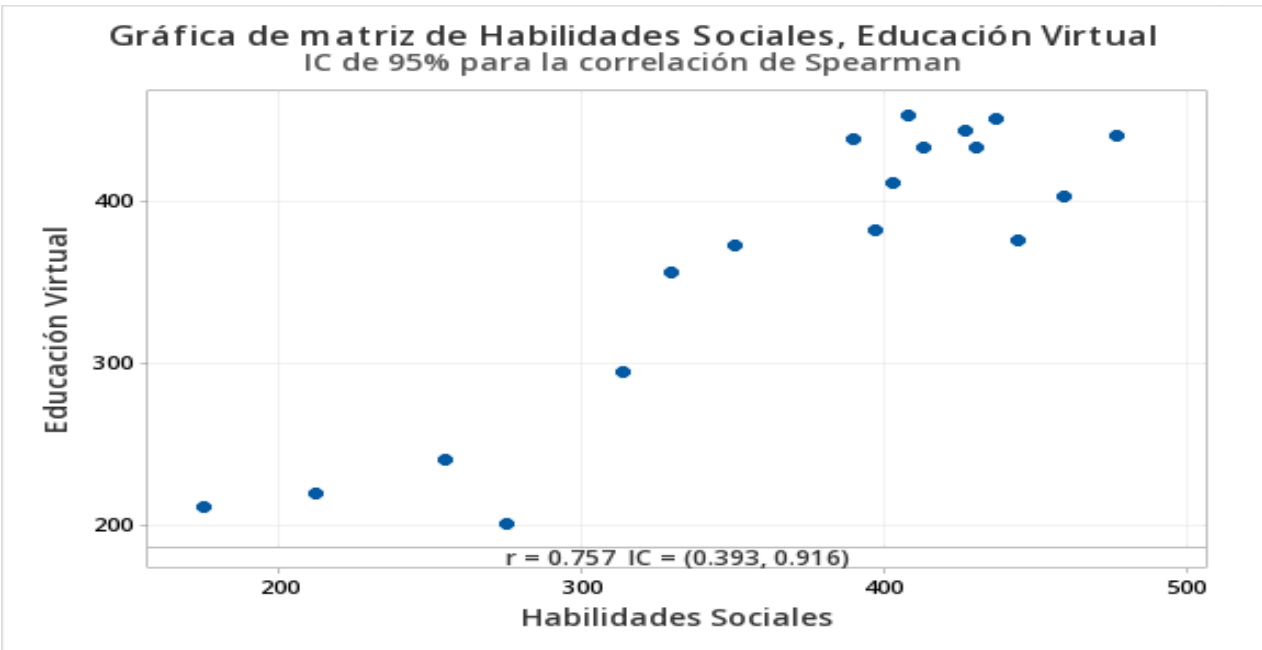

Fuente: Estudio de contextos estudiantes Universidad Técnica de Ambato

En la figura 1 se puede observar una Correlación de Spearman del 0,757 en el programa Minitab, entre las variables habilidades sociales y la educación virtual de los estudiantes de la Universidad Técnica de Ambato de la facultad de ciencias humanas y de la Educación. Lo cual muestra que hay una relación entre las habilidades sociales y la educación virtual, los estudiantes al verse obligados a cambiar su modalidad de estudios han tenido una disminución de las habilidades sociales entre pares, hay estudiantes que no han tenido la oportunidad de conocerse en persona, y solo lo han hecho por medio de las plataformas virtuales, dificultando los lazos de compañerismo.

\section{Figura 2}

Test de habilidades sociales "En busca de mi crecimiento personal pregunta 5

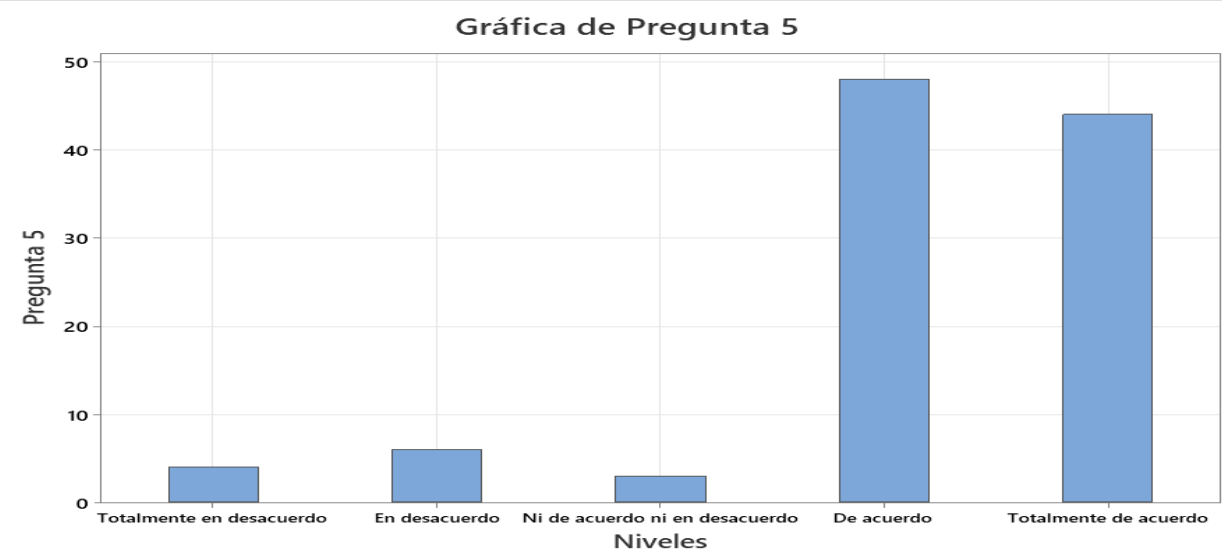

Fuente: Estudio de contextos estudiantes Universidad Técnica de Ambato 
El enunciado de la pregunta número 5 es "Conozco la importancia de las habilidades sociales en el entorno educativo", en la cual el $45,71 \%$ están de acuerdo en que es muy importante las habilidades sociales en el entorno educativo y el $41,90 \%$ de los estudiantes universitarios respondieron que están totalmente de acuerdo en la importancia de tener habilidades sociales para desarrollarse en el contexto educativo.

\section{Figura 3}

Test de habilidades sociales "En busca de mi crecimiento personal” pregunta 9

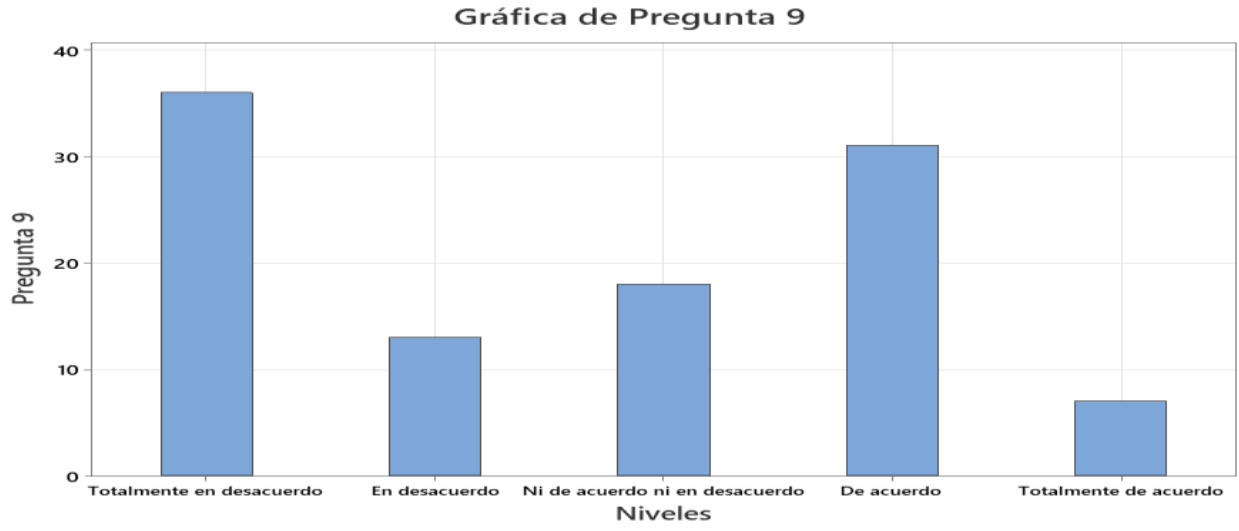

Fuente: Estudio de contextos estudiantes Universidad Técnica de Ambato

En la pregunta 9 que menciona "Me gusta tener muchos amigos para conversar con ellos", el 34,29\% responden que están totalmente en desacuerdo, lo cual evidencia que los estudiantes no están fomentando las habilidades sociales dentro del aula y el 29,52\% responden que están de acuerdo, es decir si desean contar con amigos para poder conversar con ellos lo cual se ve dificultado por la pandemia del COVID-19, que nos obliga a mantenernos aislados, lo cual en la presencialidad se daba de forma natural.

\section{Figura 4}

Encuesta sobre la educación virtual pregunta 9

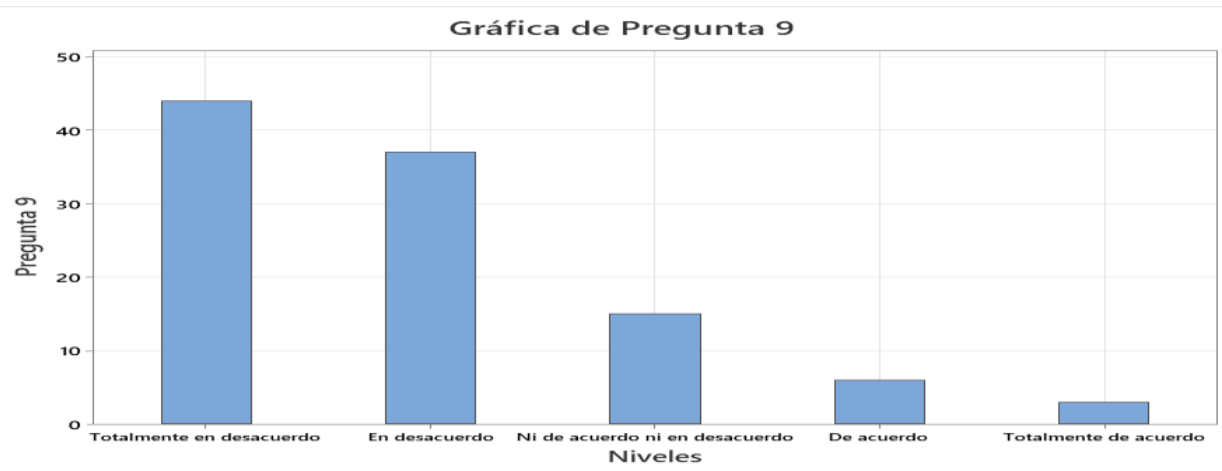

Fuente: Estudio de contextos estudiantes Universidad Técnica de Ambato 
La pregunta 9 cuestiona "¿La comunicación entre compañeros en la modalidad virtual es similar a la presencial?" a lo que el $41,90 \%$ responde que están totalmente en desacuerdo que la comunicación entre pares no es similar de manera presencial que virtual y el $35,24 \%$ respondieron que están en desacuerdo por lo que podemos inferir que las relaciones sociales son un reto entre los jóvenes universitarios en la educación virtual en comparación con la presencial, que ellos no han podido desarrollar estas habilidades de manera normal, y que en la Educación Virtual hay menos comunicación entre pares.

\section{Figura 5}

\section{Encuesta sobre la Educación virtual pregunta 17}

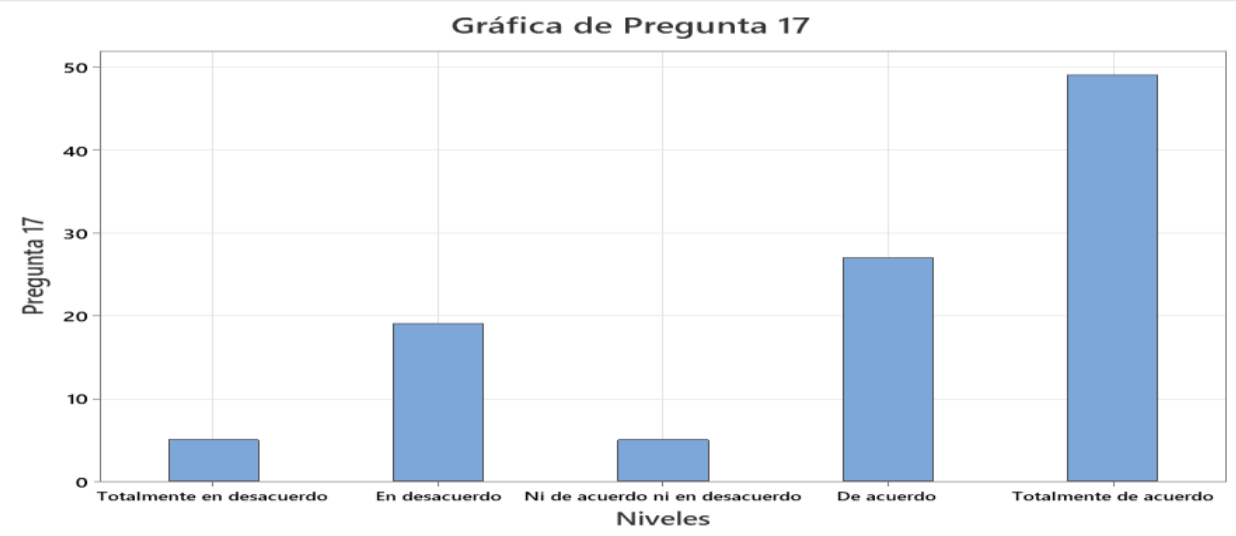

Fuente: Estudio de contextos estudiantes Universidad Técnica de Ambato

En la encuesta de educación virtual en la pregunta 17 ¿Considera que sus docentes le impulsan a usted y sus compañeros a forjar relaciones sociales entre sí?’, el 46,67\% de los estudiantes responden que están totalmente de acuerdo, que los docentes han implementado metodologías estrategias o técnicas que promuevan el desarrollen las relaciones entre pares y el $25,71 \%$ de la población encuestada respondió que estaban de acuerdo.

\section{Conclusiones}

- Las habilidades sociales se han visto afectadas por las nuevas modalidades de estudio, la falta de interacción personal entre pares y la pandemia en si ha dificultado el desarrollo de estas. Los estudiantes han desarrollado menos lasos de amistad en la modalidad virtual, hay estudiantes que no se conocen en persona, se espera que haya una adaptación a las nuevas modalidades de estudio, frente al COVID-19 que presenta nuevas variantes.

- La Educación Virtual es una modalidad de estudios que presenta ventajas y desventajas, si no se cuenta con los recursos tecnológicos necesarios para su normal desarrollo como son un ordenador, conexión a internet entre otras, 
además de un exceso de tiempo frente a un computador disminuye las relaciones personales.

- Se puede observar que hay una correlación directa entre las habilidades sociales y la educación virtual, por lo que es muy importante que las metodologías, técnicas que los docentes implementan ayuden a promover el desarrollo de las habilidades ya que esto mejora la comunicación entre los estudiantes y los prepara para un entorno laboral.

\section{Referencias Bibliográficas}

Botello, M. S., Llanos, R. M., \& Jara, Y. M. (2020). Impacto diferenciado del tiempo de formación universitaria según institución de educación media en el desarrollo de habilidades sociales. Scielo Perú, 4.

Cáceres, K. (2020). cienciamerica.uti.edu.ec. Obtenido de http://cienciamerica.uti.edu.ec/openjournal/index.php/uti/article/view/284/424

Cárdenas, A. M., \& Inacio, E. J. (2018). Desarrollo de habilidades sociales en contextos universitarios. Redalyc, 4.

Cobo, R., Vega, A., \& García, D. (20 de julio de 2020). cienciamerica.uti.edu.ec. Obtenido http://cienciamerica.uti.edu.ec/openjournal/index.php/uti/article/view/322

Coca, O. R., Merizalde, J. G., Valdiviezo, O. A., \& Sangurima, V. L. (2021). Fundamentos Pedagógicos para la enseñanza-aprendizaje de la Educación Física en Modalidad Virtual: Un reto actual. Ciencia Digital, 5.

Coronel, A. A. (2019). Laterality and reader process: correlational study. Espirales, 105.

Coronel, A. A., Suárez, P. M., Mejía, J. B., Andrade, P. A., Carrasco, E. T., \& Carpio, M. G. (2020). Habilidades sociales y agresividad en la infancia y adolescencia. Archivos venezolanos de farmacología y terapéutica, 3 .

Crisol, E., Herrera, L., \& Montes, R. (2020). dialnet.unirioja.es. Obtenido de https://dialnet.unirioja.es/servlet/articulo?codigo $=7468436$

Cueva, D. (2020). conrado.ucf.edu.cu. Obtenido de https://conrado.ucf.edu.cu/index.php/conrado/article/view/1370/1360 
García, D. N. (2019). Entorno personal de aprendizaje (PLE) basado en objetos virtuales como refuerzo en matemáticas. Ciencia Digital, 4. Obtenido de https://www.redalyc.org/articulo.oa?id=35656676003

Gavilánez, S., Cleonares, A., Nevarez, J. C., \& Sánchez, L. d. (02 de agosto de 2021). scielo.sld.cu.

Obtenido

de http://scielo.sld.cu/scielo.php?script=sci_arttext\&pid=S1990$86442021000400092 \&$ lang=es

Gil, S., \& Andreu, L. (2020). Grandes herramientas para guerreros pequeños. Catalunya: Editorial Flamboyant.

Horna-Clavo, E., Arhuis-Inca, W., \& Bazalar-Palacios, J. (2020). Relación de habilidades sociales y tipos de familia en preescolares: estudio de caso. Revista Virtual Universidad Católica del Norte, 2.

Instituto Internacional para la Educación Superior [IESAlC]. (13 de mayo de 2020). www.iesalc.unesco.org. Obtenido de http://www.iesalc.unesco.org/wpcontent/uploads/2020/05/COVID-19-ES-130520.pdf

Juca, F., Carrión, J., \& Juca, A. (02 de octubre de 2020). scielo.sld.cu. Obtenido de http://scielo.sld.cu/scielo.php?script=sci_arttext\&pid=S1990$86442020000500215 \&$ lang=es

Maximina, L. T., Isaías, R. P., Juan, A. A., \& María, R. R. (2021). Aprendizaje cooperativo $\mathrm{y}$ habilidades sociales: Universidad Nacional Jorge Basadre Grohmann. Redalyc, 7.

Ministerio de Educación Ecuador [MINEDUC]. (2020). educacion.gob.ec. Obtenido de https://educacion.gob.ec/wp-content/uploads/downloads/2020/09/CurriculoPriorizado-Costa-Galapagos-para-la-Emergencia-2020-2021.pdf

Montes, J. F., González, O. U., Legaspi, M. A., García, A. C., \& Patiño, D. E. (2018). Habilidades sociales y autoconcepto en estudiantes universitarios de la región Altos Sur de Jalisco, México. Escritos de Psicología - Psychological Writings, 4.

Morales, I. C. (2018). Las habilidades sociales y sus diferencias en estudiantes universitarios. VERTIENTES Revista Especializada en Ciencias de la Salud., 2.

Núñez, K. E., \& Zambrano, A. E. (2021). Comunicación familiar y habilidades sociales en estudiantes de educación general básica superior en una institución educativa particular de Ambato. Ciencia Digital, 4. 
Pérez, C., Suárez, R., \& Rosillo, N. (2018). www.redalyc.org. Obtenido de https://www.redalyc.org/articulo.oa?id=478055154009

Potesta, A., Ramírez, L., Alarcón, M., \& Pastor, L. (2021). revistas.pucp.edu.pe. Obtenido de https://revistas.pucp.edu.pe/index.php/anthropia/article/view/24003

Salas, P., Asún, R., \& Zúñiga, C. (2020). Construcción de un Cuestionario de Habilidades Sociales para el Contexto Académico (CHS-A). Revista Iberoamericana de Diagnóstico y Evaluación, 3.

Sousa, R., Campanari, R., \& Rodríguez, A. (2021). www.redalyc.org. Obtenido de https://www.redalyc.org/articulo.oa?id=476268269011

Tixi, D. L., Yánez, G. D., Niama, M. P., \& Costales, S. S. (2020). Impacto de la brecha digital en los procesos de enseñanza y aprendizaje en la escuela de Administración de Empresas de la Escuela Superior Politécnica de Chimborazo. Ciencia Digital, 3.

UNICEF. (04 de mayo de 2020). Covid-19: como asegurar el aprendizaje de niños son acceso a internet. https://www.unicef.org/ecuador/historias/covid-19c\%C3\%B3mo-asegurar-el-aprendizaje-de-los-ni\%C3\%B1os-sin-acceso-internet

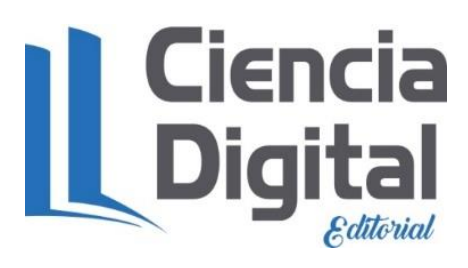


El artículo que se publica es de exclusiva responsabilidad de los autores y no necesariamente reflejan el pensamiento de la Revista Conciencia Digital.

\section{Ciencia
Digital
Edtatiol}

El artículo queda en propiedad de la revista y, por tanto, su publicación parcial y/o total en otro medio tiene que ser autorizado por el director de la Revista Conciencia Digital.
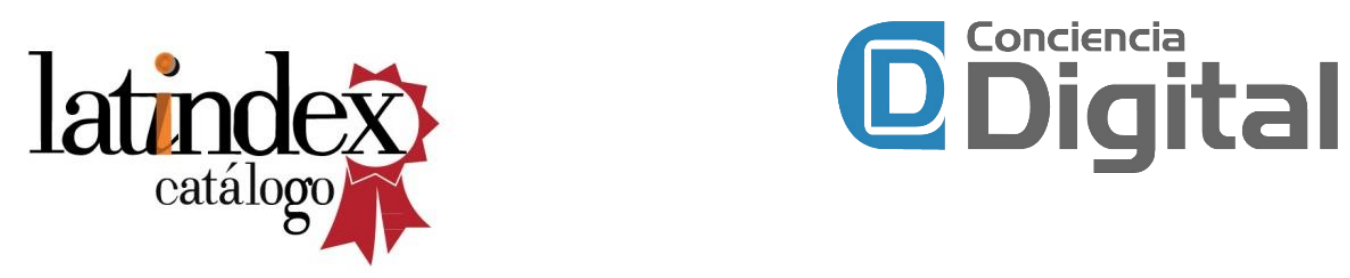

Indexaciones

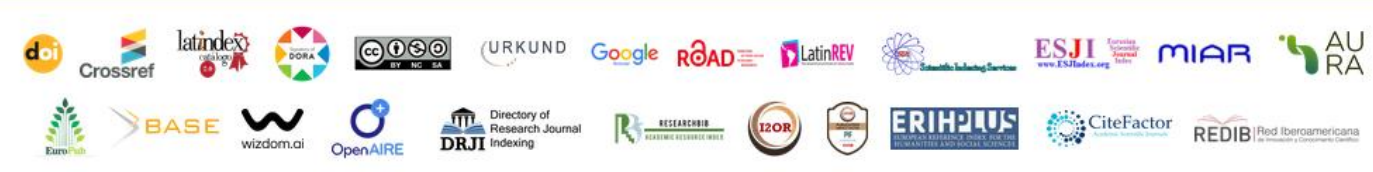

